\title{
Article \\ A MODWT-Based Algorithm for the Identification and Removal of Jumps/Short-Term Distortions in Displacement Measurements Used for Structural Health Monitoring
}

\author{
Davi V. Q. Rodrigues ${ }^{1, *}$, Delong Zuo ${ }^{2}$ and Changzhi Li ${ }^{1}$ (D) \\ 1 Department of Electrical and Computer Engineering, Texas Tech University, Lubbock, TX 79409, USA; \\ changzhi.li@ttu.edu \\ 2 Department of Civil, Environmental and Construction Engineering, Texas Tech University, \\ Lubbock, TX 79409, USA; delong.zuo@ttu.edu \\ * Correspondence: davi.rodrigues@ttu.edu
}

Citation: Rodrigues, D.V.Q.; Zuo, D.; Li, C. A MODWT-Based Algorithm for the Identification and Removal of Jumps/Short-Term Distortions in Displacement Measurements Used for Structural Health Monitoring. IoT 2022, 3, 60-72.

https://doi.org/10.3390/iot3010003

Academic Editor: Hyun-Ho Choi

Received: 31 October 2021

Accepted: 22 December 2021

Published: 24 December 2021

Publisher's Note: MDPI stays neutral with regard to jurisdictional claims in published maps and institutional affiliations.

Copyright: (C) 2021 by the authors. Licensee MDPI, Basel, Switzerland. This article is an open access article distributed under the terms and conditions of the Creative Commons Attribution (CC BY) license (https:// creativecommons.org/licenses/by/ $4.0 /)$.

\begin{abstract}
Researchers have made substantial efforts to improve the measurement of structural reciprocal motion using radars in the last years. However, the signal-to-noise ratio of the radar's received signal still plays an important role for long-term monitoring of structures that are susceptible to excessive vibration. Although the prolonged monitoring of structural deflections may provide paramount information for the assessment of structural condition, most of the existing structural health monitoring (SHM) works did not consider the challenges to handle long-term displacement measurements when the signal-to-noise ratio of the measurement is low. This may cause discontinuities in the detected reciprocal motion and can result in wrong assessments during the data analyses. This paper introduces a novel approach that uses a wavelet-based multi-resolution analysis to correct short-term distortions in the calculated displacements even when previously proposed denoising techniques are not effective. Experimental results are presented to validate and demonstrate the feasibility of the proposed algorithm. The advantages and limitations of the proposed approach are also discussed.
\end{abstract}

Keywords: Doppler radar; displacement measurement; distortion removal; IoT; jump detection; radar signal processing; structural health monitoring; smart sensor

\section{Introduction}

The average age of bridges in the United States is approximately 44 years, and $8.7 \%$ of them are considered to be in poor condition according to reports of the U.S. Department of Transportation's Federal Highway Administration [1]. On the other hand, the preservation of old historic constructions located in areas prone to seismic events or surface deformations requires their constant condition monitoring. Besides the inherent cultural heritage of historic places, there are economic, environmental, and educational benefits in preserving old buildings [2-5]. In the last decades, researchers have made efforts to develop innovative methods for monitoring the conditions of aging infrastructure, such as bridges, buildings, and stadiums [6-9]. The set of measures and routines used to retrieve information on the state of structures is called structural health monitoring (SHM). By detecting and analyzing structural damages and weaknesses based on SHM, preventive measures can be adopted to reduce the chances of fatal incidents and potential maintenance costs. The entire structural integrity process involves selecting the data acquisition system, and the number, the location, and the type of the sensors for the measurements. The main goal of SHM is to identify features in the recorded data that distinguish a near-damaged/damaged structure from an undamaged one [10-12].

Sensors that detect structural responses, such as acceleration and displacement, play an important role in SHM. Its measurements can also be used to calibrate computer-based 
models of buildings, bridges, and other structures, making the decision process during the maintenance stage more rational. Most of the current methods employed in SHM systems rely on contact sensors (e.g., accelerometers). Camera-based sensors and laser scanning systems are representatives of non-contact solutions. However, each of these have limitations. Accelerometer-based displacement measurements, which are converted from electrical signals obtained when a proof of mass is subjected to the action of a force (proportional to the measured acceleration) due to vibration, are susceptible to errors introduced by the numerical double-integration process. The performance of camerabased sensors depends on the light conditions, and poor ambient lighting can result in low-accuracy measurements and demand high computational loads for corrections. Laser vibrometers suffer from relatively high costs, and the measurement accuracy is highly affected by the superficial condition of the target.

Portable radar technology has been used in many applications that require motion measurements. For example, it has been used in animal and human vital sign monitoring, occupancy sensing, human activity classification, and security applications [13-18]. With their ability to remotely provide accurate displacement measurements, modern portable radar systems have been proved to be effective in SHM applications [19-23]. To obtain structural dynamic responses, radars take advantage of the interferometric technique, which relies on successively comparing the phase of the reflected electromagnetic waves at different times. The displacement is calculated based on the phase difference between two adjacent sampling points [24]. However, one of the drawbacks for long-term SHM using radars is that occasional low signal-to-noise ratio (SNR) of the received radio-frequency (RF) signals can introduce abrupt jumps and short-time distortions in the retrieved displacement time histories, which significantly affects the accuracy of the measurement. In the past, this issue was addressed through denoising algorithms that were able to calibrate corrupted displacements [22-24]. Nonetheless, there are still situations in which the SNR is so low, especially in the measurement of small-amplitude vibrations caused by environmental loads, that denoising-based displacement calibration techniques might not be effective.

The accuracy of the retrieved phase-demodulated measurements depends on several factors, including multipath effects, the instantaneous magnitude of the noise level, the carrier frequency of the transmitted radar signal, the amplitude of the observed reciprocal motion, the power level of the transmitted signal, the gain of the baseband amplifiers, and the directivity of the transmitting and receiving antennas. However, the major factor that impacts the accuracy of the radar-based displacement measurements is the SNR of the received microwave signals. For example, during the continuous monitoring of the mast arm deflection of a traffic signal support structure, which will be used as an example of application in this study, the amplitude of the motion has been found to be too small during some short instants. This has resulted in either sudden jumps and/or shorttime distortions in the displacement measurements that cannot be effectively removed by denoising techniques. The main contribution of this paper is to propose an automated scheme that relies on the multi-resolution analysis of the maximal overlap discrete wavelet transformation (MODWT) of the radar-based phase-demodulated measurements to identify jumps and/or short-term distortions and correct the displacements even when denoising strategies are not effective.

Other time-varying spectral analysis-based approaches, such as those that use empirical mode decomposition (EMD), variational mode decomposition (VMD, and synchrosqueezed wavelet transforms (SWT) may also be considered for the design of algorithms for the automated detection and removal of sudden changes/short-distortions on phase-demodulated Doppler radar data [25-28]. The application of empirical basis decomposition methods, such as EMD and VMD to a time series, produces a set of intrinsic modes and a residual signal without the need of a priori basis system. Particularly, EMD-based techniques generate the so-called intrinsic mode functions. When the EMD-based techniques are associated with the Hilbert transform, the resulting combination is known as Hilbert-Huang transform (HHT), which has been used for radar-based vital sign detection, 
human activity recognition, and dam-age assessment [29-32]. Although EMD-based algorithms might be utilized for the analysis of non-stationary/nonlinear signals and have demonstrated its robustness and validity for various applications, it still lacks a reliable and generally accepted theoretical foundation, which would allow for convergence proofs and systematic optimization of the method [33]. Furthermore, EMD-based technique may generate mode mixing during decomposition, which happens when a particular intrinsic mode function possesses components of different frequencies $[28,34,35]$. On the other hand, synchrosqueezing transforms (SST) is a recently developed time-frequency analysis method recommended for the study of multicomponent signals with oscillating modes [36]. It is a combination of a time-frequency decomposition method (wavelet packet transform, curvelet transform, short-time Fourier transform, or continuous wavelet transform) and a reassignment step. Researchers frequently employ it as an alternative to EMD-based algorithms, since SST-based techniques benefit from having a closed mathematical derivation, which allows for better understanding and the possibility of refinement based on the desired application $[37,38]$. SST-based algorithms have been used for sinusoidal noise removal in partial discharge measurements, seismic time-frequency analysis for hydrocarbon detection, and for the faulty diagnosis of a wind turbine planetary gearbox under nonstationary conditions [39-41]. SST-based algorithms also rely on a linear time-frequency representation associated with a fixed time-frequency resolution, which is given by a general window or wavelets [42,43]. In this work, the proposed algorithm is based on the multiresolution analysis of the MODWT of the deflections measured by a Doppler radar. The MODWT can be understood as a modified version of the discrete wavelet transform (DWT) with the advantages of being able to handle measurements of any sample size and being invariant to circular shifting of the time series. Furthermore, both discrete wavelet-based transforms can be employed as a variance estimator. However, the MODWT-based wavelet variance estimator is asymptotically more efficient than the equivalent DWT-based variance estimator [44]. The reason why the MODWT is preferred over the wavelet-based synchrosqueezed transform is because the latter is evaluated using the continuous wavelet transform (CWT) of the non-stationary/nonlinear signals. Although the CWT also preserves time shifts and time scaling, it also adds excessive redundancy, and it is computationally intensive [45]. Due to the translation invariant for the wavelet coefficients and the scale invariance, all decomposition levels of the MODWT feature the same time resolution without phase distortion, which enables a comparison between the original time series and its decomposition. In contrast to EMD-based methods, MODWT-based algorithms offer more flexibility; since they can be easily adapted to the intended application, it can be readily understood because it is based on a closed mathematical formulation, and it does not require a preliminary investigation based on either empirical estimation or drastic changes of the sifting procedure [46]. Since part of the statistical properties of the retrieved displacements corrupted by sudden jumps/short-term distortions are known, empirical mode decomposition or even empirical wavelet transforms would not be better choices. As a matter of fact, empirical transformations would be less efficient as they initially need to adaptively decompose the original signal without previous knowledge of its statistical properties.

In this work, the effectiveness of the novel MODWT-based algorithm for the identification and removal of jumps/short-time distortions is evaluated. Although the presented results are based on the long-term monitoring of a traffic signal structure conducted by a Doppler radar, the proposed algorithm can be readily employed for other vibration measurements by radars operating with different waveforms (e.g., frequency-modulated continuous-wave radars, stepped-frequency continuous-wave radars). The rest of this paper is organized as follows. In Section 2, the theory for SHM based on Doppler radar and the multi-resolution analysis of the MODWT coefficients is reviewed. In Section 3, the proposed wavelet-based algorithm is detailed. The experimental results are discussed in Section 4. Finally, the conclusions are drawn in Section 5. 


\section{Theory}

\subsection{Nonlinear Phase-Demodulation Procedure}

Figure 1 exhibits the block diagram for the long-term SHM of a traffic signal structure using Doppler radar. A continuous-wave $(\mathrm{CW})$ microwave signal is transmitted towards the ground. After the returned RF echo is mixed with a replica of the transmitted microwave signal, the baseband in-phase and quadrature phase signals $I(t)$ and $Q(t)$ are obtained. The normalized outputs $I(t)$ and $Q(t)$ can be represented as

$$
\begin{gathered}
I(t)=A_{I} \cdot \cos (4 \pi x(t) / \lambda+\Delta \phi(t))+d c_{I} \\
Q(t)=A_{Q} \cdot \sin (4 \pi x(t) / \lambda+\Delta \phi(t))+d c_{Q}
\end{gathered}
$$

where $A_{I}$ and $A_{Q}$ are the corresponding amplitudes of the quadrature signals, $d c_{I}$ and $d c_{Q}$ are the dc offsets, $x(t)$ is the reciprocal motion, $\lambda=c / f$ is the free-space wavelength, $f$ is the microwave frequency of the transmitted signal, and $\phi(t)$ denotes the phase associated with the round-trip added to the residual phase noise of the voltage-controlled oscillator. The $I / Q$ signals must be preconditioned before the application of the nonlinear phasedemodulation procedure $[47,48]$. After the use of a circle fitting algorithm to correct $I / Q$ amplitude imbalances and to calibrate the dc offsets, the resulting normalized complexvalued baseband signal can be written as

$$
s(t)=\overline{I(t)}+j \overline{Q(t)}=\sum_{n=-\infty}^{\infty} B_{n}(4 \pi \delta / \lambda) \cdot \exp \left(j n 2 \pi f_{0} t+\Delta \phi(t)\right)
$$

where $\overline{I(t)}$ and $\overline{Q(t)}$ are the normalized baseband signals, $f_{0}$ is the dominant frequency of the relative motion that vibrates with a periodic time-varying amplitude $\delta\left(x(t)=\delta \cdot \sin \left(2 \pi f_{0} t\right)\right)$, and $B_{n}$ is the $n$-th order Bessel function of the first kind. The Doppler radar-based displacement can be calculated as follows:

$$
d(t)=(\lambda / 4 \pi) \cdot \arctan (\operatorname{Im}[s(t)] / \operatorname{Re}[s(t)])
$$

which clearly indicates that an arctangent-based demodulation approach is required for the accurate displacement reconstruction. The sampled version of (4) can be written as $d(n)=(\lambda / 4 \pi) \times \arctan \left(\frac{\operatorname{Im}[s(n)]}{\operatorname{Re}[s(n)]}\right)$, where $n=0 . \Delta t, 1 . \Delta t, 2 . \Delta t, \ldots,(N-1) . \Delta t$, with $N$ being the digitized data length and $\Delta t=1 / f_{s}$ the sampling interval for a sampling frequency equals to $f_{s}$. Since the arctangent function has a domain limitation of $(-\pi / 2$, $\pi / 2)$, phase ambiguities must be addressed before the application of the arctangent transformation. Phase ambiguities might happen when the amplitude of the reciprocal motion is over a quarter wavelength. As a consequence, the length of the $I / Q$ trajectory exceeds $\pi$ and discontinuities appear during the arctangent demodulation [24,47]. In fact, phase ambiguity, which constitutes a critical issue in radar interferometric sensing of reciprocal motion, may still exist even when the amplitude motion is less than a quarter wavelength because the $I / Q$ trajectory might sit across quadrants [48].

\subsection{Theory of the Multiresolution Analysis for MODWT Coefficients}

The wavelet-based multi-resolution analysis of measurements contaminated with jump/short-term distortions can provide the onset times of jump/short-term disturbances on the retrieved radar displacement with relatively high time resolution. In this work, the maximum overlap discrete wavelet transform (MODWT), which is a version of the discrete wavelet transform (DWT), was utilized because the MODWT coefficients are not affected by the starting point of the time series or the size of the recorded data [49-53]. 


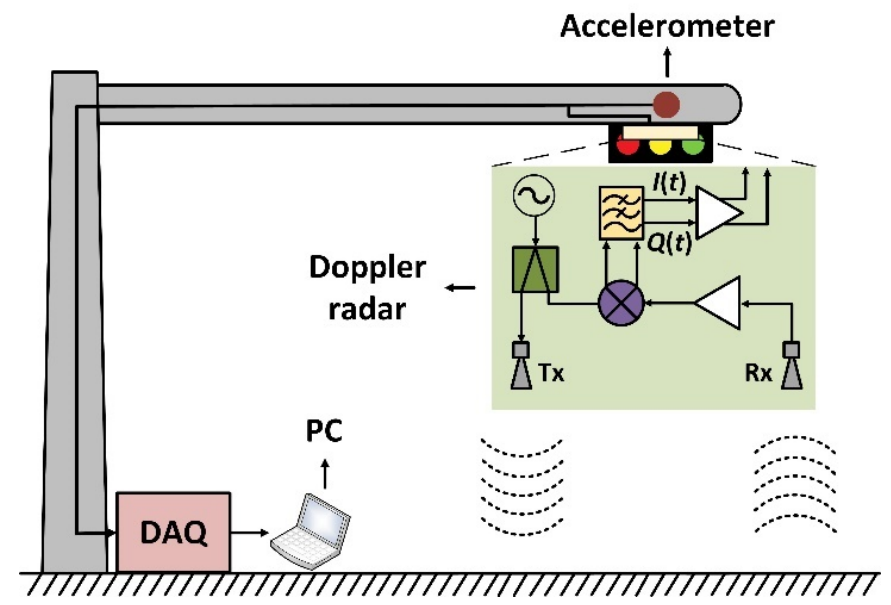

Figure 1. Block diagram for the structural health monitoring of a traffic signal structure using a Doppler radar.

In the discussion to follow, boldface lowercase letters and boldface uppercase letters will denote vectors and matrices defined for $n=0 . \Delta t, 1 . \Delta t, 2 . \Delta t, \ldots,(N-1) . \Delta t$, respectively. The $i$-th component of an $N$-element vector $d$ will be refer to as $d_{i}$. Consider the sampled value of the displacement organized as a vector $d=\left\{d_{0} d_{1} d_{2} \ldots d_{N-1}\right\}$ to eliminate the sensitivity of the DWT to the starting point of $d$, which is caused by the downsampling of the outputs from the scaling and the wavelet filters, one should apply the DWT to $d$ and the circularly shifted column vector $S \boldsymbol{d}=\left\{d_{2} d_{3} d_{4} \ldots d_{N-1} d_{1}\right\}$, where $S$ is an $N \times N$ circular shift matrix, and then concatenate the set of resulting coefficients to create the MODWT coefficients. However, to recognize the fast ruptures of the displacement measurements with high time resolution, particularly the starting point of sudden jumps and/or short-term disturbances, the multi-resolution analysis (MRA) of the MODWT coefficients must be employed because the MODWT does not provide time-aligned detailed features. The MRA of the MODWT can be considered as zero-phase bandpass filtering of the original signal, i.e., the detailed features on several scales will be time-aligned $[53,54]$.

To decompose a finite time-series signal using the MODWT to $J$ levels, one must apply $J$ pairs of wavelet (high-pass) $\widetilde{\boldsymbol{h}}_{j, l}$ and scaling (low-pass) filters $\widetilde{\boldsymbol{g}}_{j, l}$. The filtering operation at the $j$-th level yield a set of wavelet and scaling coefficients as shown below:

$$
\begin{aligned}
& \widetilde{w}_{j, t}=\sum_{l=1}^{L_{j}-1} \widetilde{h}_{j, l} \cdot d_{t-l \bmod N} \\
& \widetilde{v}_{j, t}=\sum_{l=1}^{L_{j}-1} \widetilde{g}_{j, l} \cdot d_{t-l \bmod N}
\end{aligned}
$$

where $t=0,1,2, \ldots, N-1, \widetilde{\boldsymbol{h}}_{j, l}$, and $\widetilde{\boldsymbol{g}}_{j, l}$ are the $j$-th level MODWT wavelet and scaling filters. $\widetilde{w}_{j, t}$ and $\widetilde{v}_{j, t}$ are the wavelet and scaling coefficients, and $\alpha \bmod \beta$ stands for $\alpha$ modulo $\beta$ from modular arithmetic [55-57]. Each of the MODWT filters has width $L_{j}=\left(2^{j}-1\right)(L-1)+1$, where $L$ is the width of the base filter $(j=1)$ and constitute a set of scale-dependent differencing and averaging operators, respectively. Additionally, they can be interpreted as the stretched versions of the base filters $(j=1)$. For example, the nonzero portion of the Haar MODWT base filter is defined as $\widetilde{\boldsymbol{h}}_{1, l}=\widetilde{\boldsymbol{h}}_{l}=\{1 / 2-1 / 2\}$ and the nonzero portion of the corresponding MODWT scaling base filter is $\widetilde{\boldsymbol{g}}_{1, l}=\widetilde{\boldsymbol{g}}_{l}=\{1 / 21 / 2\}$.

Assuming that $\widetilde{\boldsymbol{h}}_{j, l}^{\circ}$ represents $\widetilde{\boldsymbol{h}}_{j, l}$ periodized to length $N$, and $\widetilde{\boldsymbol{g}}_{j, l}^{\circ}$ is $\widetilde{\boldsymbol{g}}_{j, l}$ periodized to length $N$, the evaluated displacement can be cast as the following linear combination:

$$
\begin{gathered}
d=a_{J}+\sum_{k=1}^{J} \boldsymbol{b}_{\boldsymbol{k}} \\
\boldsymbol{a}_{\boldsymbol{J}, t}=\sum_{l=0}^{N-1} \widetilde{g}_{J, l}^{\circ} \widetilde{v}_{J, t+l \bmod N}
\end{gathered}
$$




$$
\boldsymbol{b}_{k, t}=\sum_{l=0}^{N-1}{\widetilde{h^{\circ}}}_{k, l} \widetilde{w}_{k, t+l \bmod N}
$$

where $k=1,2,3, \ldots, J$ is the scale number, $J$ is the number of levels for the wavelet decomposition, usually defined as the nearest integer less than or equal to $\log _{2}(N), \boldsymbol{a}_{\boldsymbol{J}}$ is the approximation vector, and $\boldsymbol{b}_{\boldsymbol{k}}$ are the detail vectors at successive scales. The detail vectors are calculated at each level. In contrast, the scaling vector is only produced for the final level. In other words, the MRA of the MODWT returns the projections of $\boldsymbol{d}$ onto the various wavelet subspaces and final scaling subspace. The detail vectors $v_{l}$ contain high-frequency content, while $\boldsymbol{a}_{\boldsymbol{J}}$ is the coarse scale approximation (low-frequency content) of the estimated displacement for each scale $J$ [49-54]. This paper does not cover all the aspects of MODWT and its MRA derivations. Interested readers are recommended to refer to [49], where a complete description of the calculations required to evaluate the MRA of the MODWT of a time-domain signal are explained.

\section{Proposed MODWT-Based Algorithm}

Figure 2 details the proposed wavelet-based approach for the identification and removal of jumps and/or short-time disturbances in contaminated phase-demodulated measurements. The distorted displacement (disp), the sampling frequency $(F s)$, the window length in seconds used on the Find local maxima subroutine ( $T$ ), and the maximum duration of the short-time disturbances are the inputs required by the proposed algorithm. Since the sudden jumps and short-term discontinuities would better match short width wavelets, the Haar wavelet was chosen as the basis wavelet. Additionally, the appearance of jumps and short-term distortions would add high-frequency spectral components to the analyzed displacement measurement. Therefore, the detail features of the spectral decomposition of the displacement measurements were the focus of our study. The close time synchronization between the $i$-level detail vector and the contaminated displacement measurement for the sudden occurrences of jumps/short-term disturbances plays the key role in choosing the best detail vector.

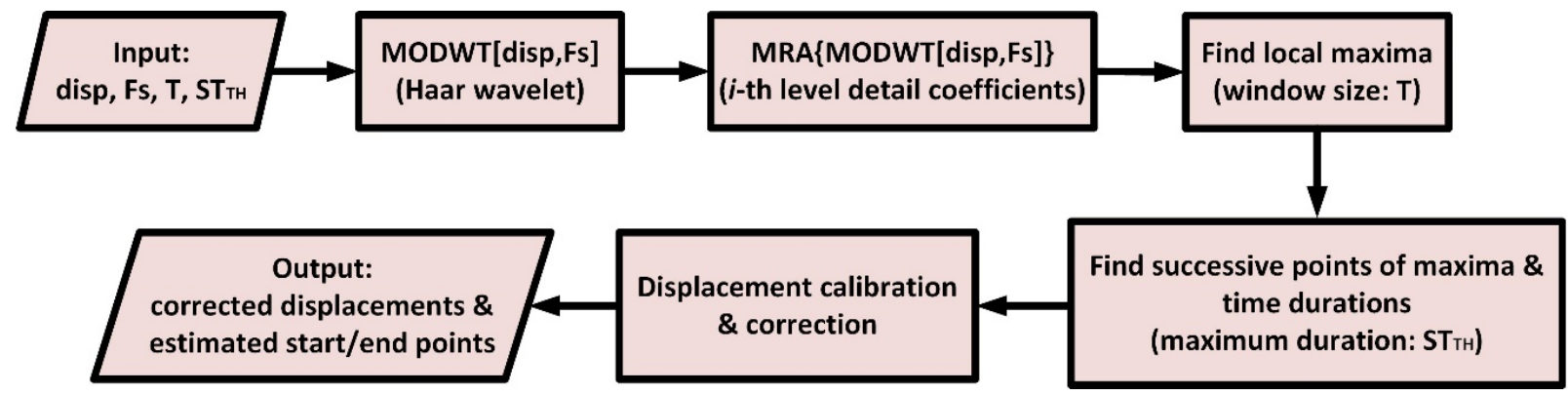

Figure 2. Flowchart of the MODWT-based algorithm.

In the algorithm illustrated in Figure 2, the MODWT of the total displacement (disp) is initially calculated. Then, the MRA for the MODWT coefficients is evaluated. In the Find local maxima subroutine, the amplitude threshold for the detection of jump/short-term disturbance is assigned as three times the root-mean-square (RMS) value of the normalized amplitude of the $i$-th level detail coefficients to reduce the number of false positive detections. This value was determined after the extensive analysis of several datasets recorded over approximately three months. To accurately identify the points of local maxima, which are the starting points of abrupt changes on the analyzed displacement, the Find local maxima subroutine is applied to $T$-seconds-long portions of displacement measurements, and a vector with the corresponding times of the jumps/short-term disturbances is created. Since an objective is to remove potential short-term disturbances, distortions that follow the sudden jumps are also recognized by a subroutine that looks for successive points of local maxima. Based on the fact that the mean value of a sinusoidal evolution over time is con- 
stant and by taking advantage of the starting points for the jumps/short-term disturbances, a displacement calibration subroutine is applied to dis $p$ to remove potential deviations on the baseline level of the measurements. Finally, a displacement correction subroutine is applied to the portion of the measurements with sudden jumps and/or distortions. To eliminate contaminated measurements that may lead to wrong structural assessments, their amplitude values are set to zero, which is assumed as the mean value of the sinusoidal measurement. Furthermore, since the time location of the jumps/short-term disturbances might not coincide with the actual time location of the abrupt changes, the vector with the corresponding time locations of the jumps/short-term disturbances is updated by adding the period of the observed motion $\left(1 / f_{0}\right)$ to each entry. The entries of the time-localization vector will be used to automatically localize the non-distorted radar-based measurements that can be employed on further structural analysis.

\section{Experimental Results}

To investigate the effectiveness of the proposed automated jump/short disturbance identification and removal algorithm, a traffic signal structure, which consists of a $12 \mathrm{~m}$ horizontal mast arm and a $6 \mathrm{~m}$ vertical pole, was used as a testbed. The deflections of the horizontal mast arm induced by random environmental loads were continuously monitored for several weeks by a Doppler radar housed in a plastic enclosure and mounted near the tip of the mast arm. In the meantime, the mast's vibration was also measured by a conventional tri-axial DC-response accelerometers of $\pm 4 \mathrm{~g}$ measurement range that was mounted at the same axial location of the radar on the arm. Both the $I / Q$ channels of the radar and the channels of the accelerometer were sampled at $200-\mathrm{Hz}$ by a data acquisition system (DAQ) consisting of National Instrument NI-9239 voltage input modules on a CompactRIO platform. Figure 3 exhibits the experimental setup.

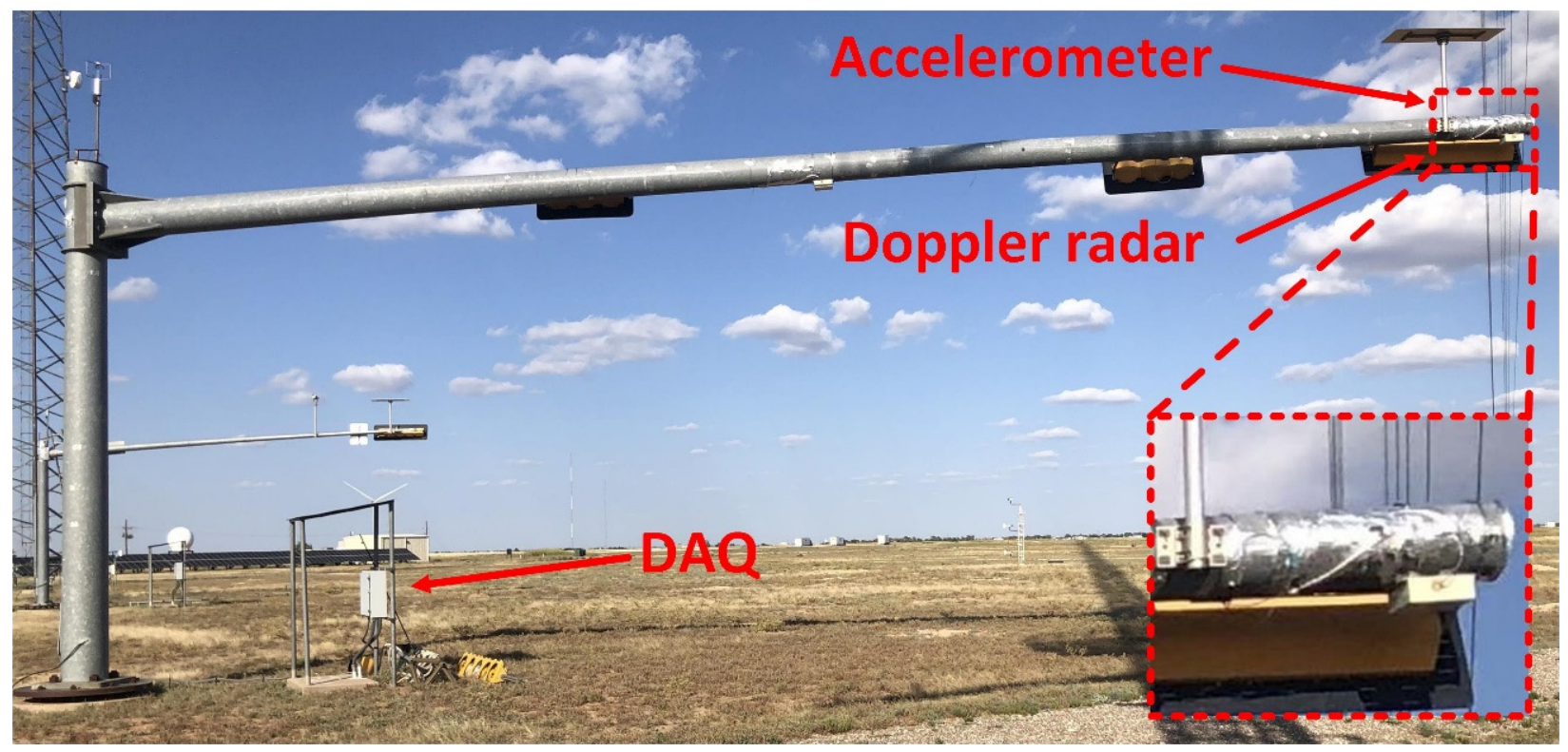

Figure 3. Experimental setup for the remote long-term SHM of a traffic signal structure based on Doppler radar. The inset shows the radar enclosure.

After analyzing several recorded datasets, it was determined that the recovered baseband signals related to the motion amplitudes of $\sim 2 \mathrm{~cm}$ were the ideal candidates to validate the proposed algorithm. Since the amplitude of the motion of the tip of the mast was $2 \mathrm{~cm}$, the SNR of the recovered baseband signals was considerably low. Therefore, sudden jumps appeared on the phase-demodulated displacement measurements. The Adaptive Joint Signal Processing Algorithm (AJSPA) and the Adaptive Lowpass Filtering Algorithm (ALFA), which are denoising strategies proposed by the authors in a previous study, were then 
applied to the baseband signals [22]. The SNR was so low that the abrupt jumps were not successfully removed by the denoising techniques. In addition, short-term distortions were seen in the demodulated radar data. Figure 4 shows a 125-s radar-based displacement after applying the AJSPA to the baseband signals, and the reference, which is the displacement calculated by numerically integrating the corresponding accelerometer measurement twice and high-pass filtering the result of each step of integration with a cutoff frequency of $0.5 \mathrm{~Hz}$. Four abrupt jumps and two short-term distortions can be visually identified around the instants $30.00 \mathrm{~s}, 44.05 \mathrm{~s}, 64.74 \mathrm{~s}, 67.47 \mathrm{~s}, 81.74 \mathrm{~s}, 82.34 \mathrm{~s}, 98.15 \mathrm{~s}$, and $117.89 \mathrm{~s}$. The RMS error (using the accelerometer measurement as the reference) for the estimated displacement is $1.14 \mathrm{~cm}$. The proposed MODWT-based algorithm introduced in Section III was programmed in MATLAB and applied to the analyzed displacement. The window length $T$ was chosen to be $5 \mathrm{~s}$. Although the standard MODWT algorithm implements the circular convolution in the time domain, the wavelet and scaling filter coefficients at level $j$ $(j>1)$ were evaluated by the circular convolutions in the frequency domain by taking the inverse discrete Fourier transform of the products of the discrete Fourier transforms. Due to the close time synchronization between the 5th-level detail vector and the displacement measurement contaminated with sudden jumps and/or short-term distortions, the coefficients of the 5-th level detail vector were chosen. In this work, distortions that appear for up to $3 \mathrm{~s}(\mathrm{STTH}=3 \mathrm{~s})$ are considered short-term disturbances. Figure 5 shows the MODWT 5th-level detail decomposition associated with the radar-based measurement shown in Figure 4. The points of local maxima are highlighted, and they can be easily seen around 29.53 s, 43.69 s, 64.36 s, 66.93 s, 81.29 s, 81.75 s, 97.72 s, and 117.52 s. It should be noted that they are closely separated from the actual starting points of the sudden jumps/short-term distortions highlighted in Figure 4. In fact, the MODWT-based technique anticipates the time instants for the commencement of the disturbances in less than $\sim 1 \mathrm{~s}$.

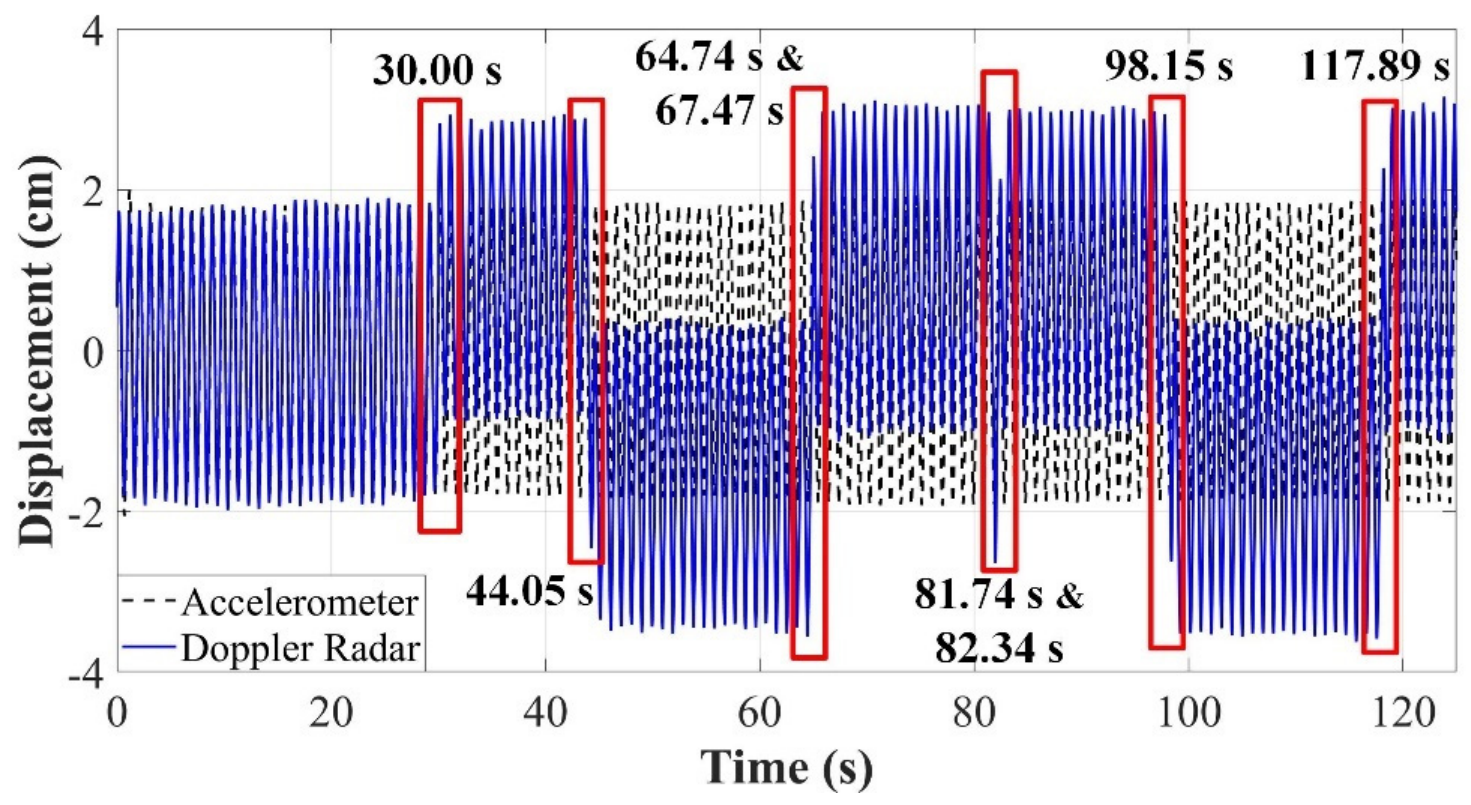

Figure 4. Displacement retrieved by a Doppler radar. The discontinuities are highlighted.

Since the changes can be clearly seen on the baseline of the displacement, one can argue that a time-domain-based strategy would be more effective for the identification and removal of jumps/short-term disturbances in phase-demodulated signals. Figure 6 reveals the displacement measurement obtained after applying a corresponding timedomain-based algorithm (TDBA) to the deteriorated measurement shown in Figure 4 . The main difference between the TDBA and the proposed MODWT-based technique is that the occurrence of a sudden jump is associated with the absolute difference between the baselines of the first half and the second half of the demodulated displacement for each 
window length $\mathrm{T}$, which is programmed as $5 \mathrm{~s}$ as it was programmed for the MODWTbased strategy. The TDBA would flag the presence of jumps/distortions if the absolute difference between the mean of the two halves of the displacement for each window was larger than $1 \mathrm{~mm}$. As shown in Figure 6, the corresponding time-domain-based approach method showed the presence of discontinuities at several windows, including portions of the displacement that did not have any distortion. Due to the lack of time resolution of the TDBA, the exact locations of the jumps/distortions could not be provided.

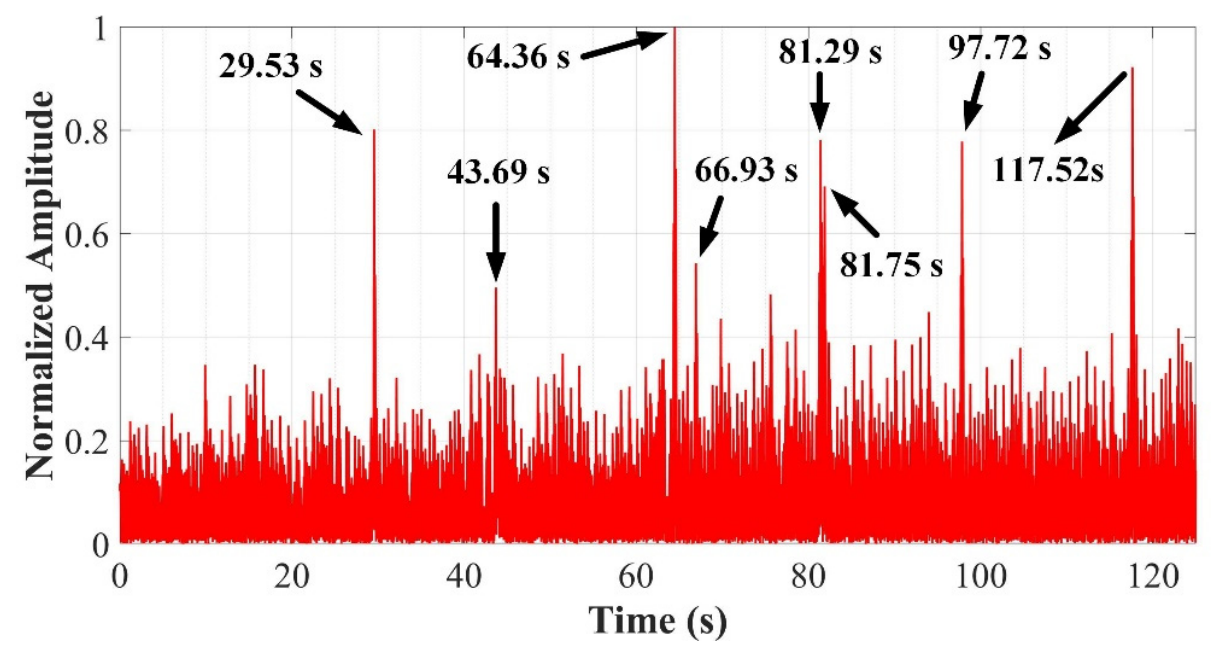

Figure 5. MODWT 5th-level detail decomposition using Haar wavelet of the deteriorated radar-based measurement.

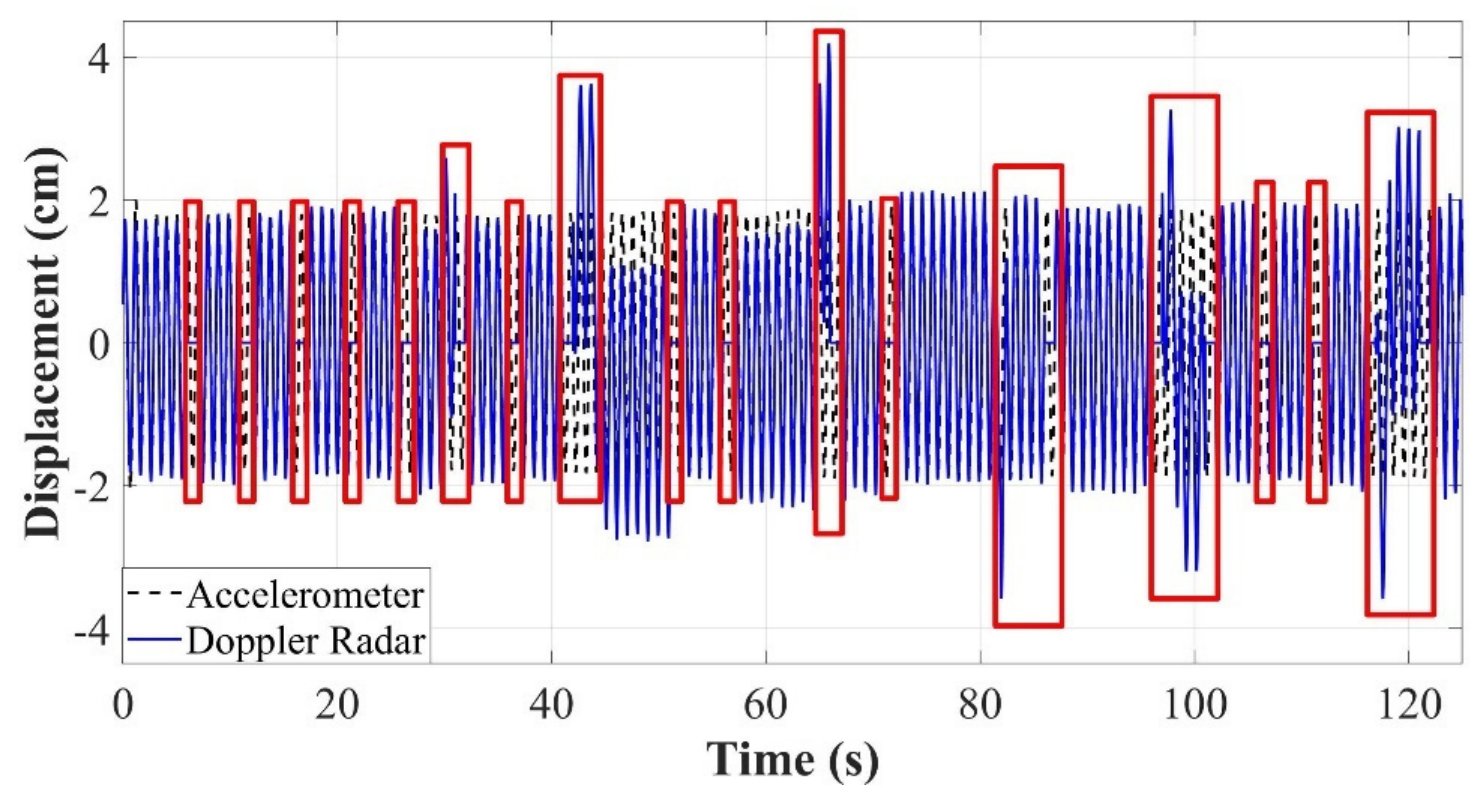

Figure 6. Displacement corrected by a time-domain-based approach. The distortions are highlighted.

The MODWT-based technique was also applied to the analyzed displacement. Figure 7 illustrates the corrected measurement after the application of the proposed jump/shortdistortion identification and removal technique. By employing a MODWT-based approach, the exact locations of the jumps/distortions were provided due to the localization property of the wavelet-based approach. The amplitude of the portions of demodulated Doppler radar data identified as deteriorated were set to zero. Four abrupt jumps and two shortterm distortions were correctly identified. The vector with the corresponding time locations of the jumps/short-term disturbances was updated by adding the period of the observed 
motion ( 1 s) to each entry, and the starting/end points of the corrected portions of the recovered radar-based displacement were successfully recognized.

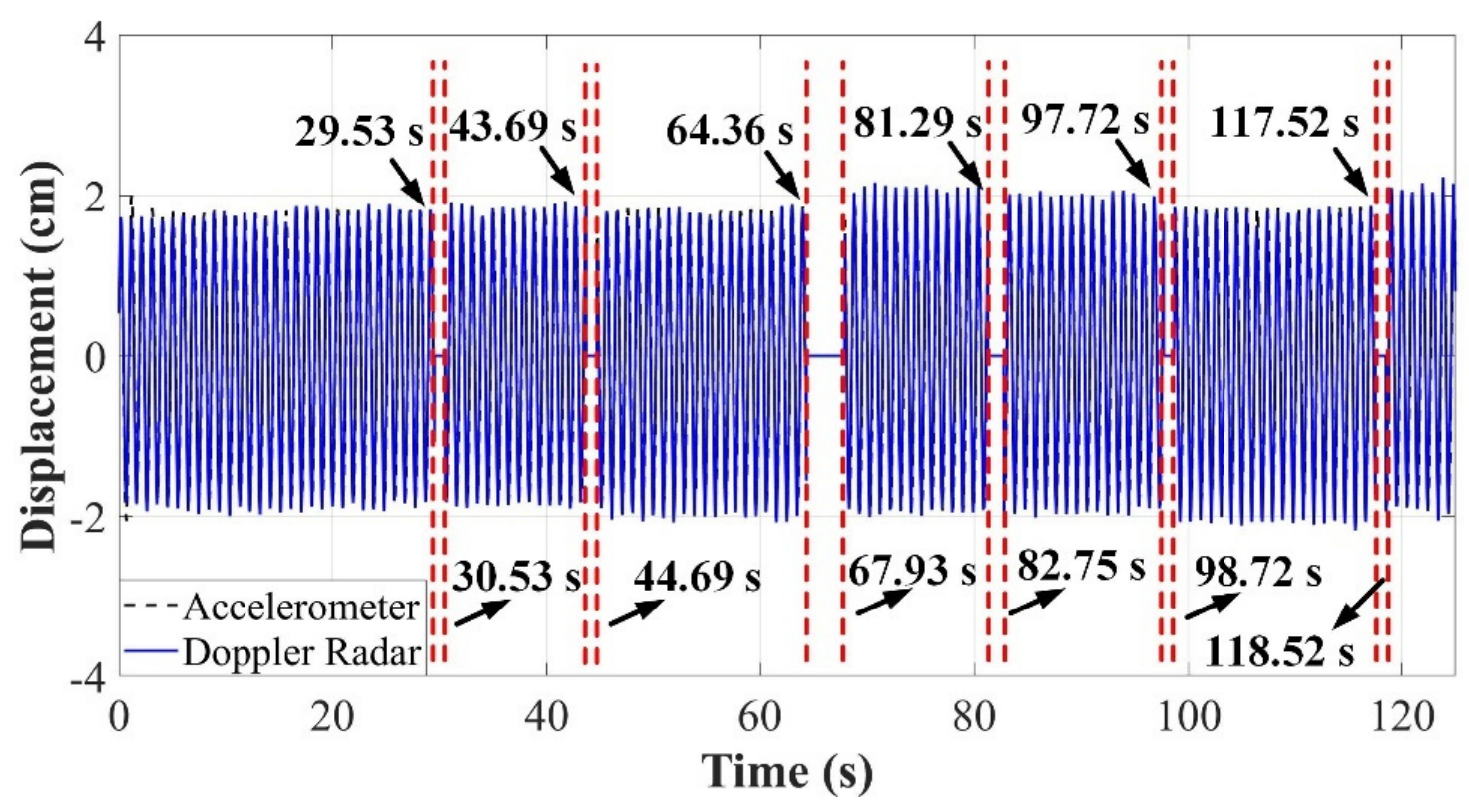

Figure 7. Displacement corrected by the proposed algorithm. The corrected portions of the retrieved radar-based displacement were successfully recognized.

To further investigate the robustness of the proposed method, several contaminated datasets were analyzed as depicted in Table 1. Due to low SNR for the recorded $I / Q$ baseband signals at times, various jumps and/or short-term distortions were recognized at the phase-demodulated displacement recorded at the tip of the mast. Therefore, both the AJSPA and the ALFA were applied to 11 different datasets collected after monitoring the traffic signal structure for almost a month. The length of the datasets varies from $40 \mathrm{~s}$ to $500 \mathrm{~s}$. The accuracy of the free vibration displacement measurements is evaluated by comparing Doppler radar-based measurements and the accelerometer-based measurements through the calculation of the RMS error for each data set. Since the denoising techniques were not able to eliminate the disturbances, Haar wavelet-based algorithm was applied to them to identify and remove the deteriorated portions of phase-demodulated radar measurements. As is shown on Table 1, the proposed strategy was able to correctly identify and remove the distortions, reducing the RMS errors for each analyzed dataset. No correlation between the calculated RMS errors and the length of the analyzed time series was identified.

Table 1. Accuracy of the radar-based measurements.

\begin{tabular}{ccccc}
\hline Data Set & Length (s) & $\begin{array}{c}\text { Denoising } \\
\text { Algorithm }\end{array}$ & $\begin{array}{c}\text { Denoising Algorithm } \\
\text { RMS Error (cm) }\end{array}$ & $\begin{array}{c}\text { Proposed Approach } \\
\text { RMS Error (cm) }\end{array}$ \\
\hline 1 & 125 & AJSPA/ALFA & $1.14 / 1.20$ & $0.27 / 0.22$ \\
\hline 2 & 500 & AJSPA & 1.89 & 0.25 \\
\hline 3 & 50 & ALFA & 0.73 & 0.28 \\
\hline 4 & 100 & AJSPA/ALFA & $1.35 / 0.65$ & $0.37 / 0.30$ \\
\hline 5 & 101 & AJSPA & 1.19 & 0.25 \\
\hline 6 & 40 & AJSPA/ALFA & $0.86 / 0.74$ & $0.38 / 0.31$ \\
\hline 7 & 100 & AJSPA & 1.19 & 0.28 \\
\hline 8 & 50 & AJSPA & 0.87 & 0.39 \\
\hline
\end{tabular}


Table 1. Cont.

\begin{tabular}{ccccc}
\hline Data Set & Length (s) & $\begin{array}{c}\text { Denoising } \\
\text { Algorithm }\end{array}$ & $\begin{array}{c}\text { Denoising Algorithm } \\
\text { RMS Error (cm) }\end{array}$ & $\begin{array}{c}\text { Proposed Approach } \\
\text { RMS Error (cm) }\end{array}$ \\
\hline 9 & 250 & AJSPA & 2.01 & 0.30 \\
\hline 10 & 200 & AJSPA & 1.28 & 0.26 \\
\hline 11 & 100 & AJSPA/ALFA & $0.40 / 0.76$ & $0.25 / 0.35$ \\
\hline
\end{tabular}

\section{Conclusions}

The identification of jumps and/or short-term distortions on radar-based displacements is of paramount importance for the effective application of radar-based SHM. The presence of disturbances on the phase-demodulated radar measurements can cause incorrect estimation of structural parameters and lead to false alarms. In this paper, we propose a novel automated jump/short-term distortion identification and removal algorithm using MODWT-based spectral analysis. A series of full-scale experiments demonstrated that the wavelet-based approach has significantly better performance than the time-domain-based technique and can be successfully applied to distorted displacements even when denoising techniques are not effective. Although a traffic signal structure was used as a testbed, the proposed method may be employed in long-term SHM of other structures, such as buildings and bridges, that rely on the analysis of displacement measurements. Future work will focus on the impact of the environment variations, such as temperature and humidity, on the accuracy of radar displacement measurements used for long-term SHM.

Author Contributions: Conceptualization, D.V.Q.R., methodology, D.V.Q.R., D.Z. and C.L.; validation, D.V.Q.R.; writing—original draft preparation, D.V.Q.R.; writing—review and editing, D.Z. and C.L.; supervision, C.L.; project administration, D.Z. and C.L.; funding acquisition, C.L. All authors have read and agreed to the published version of the manuscript.

Funding: The authors would like to acknowledge funding support from National Science Foundation (NSF) under grants ECCS-1808613.

Conflicts of Interest: The authors declare no conflict of interest.

\section{References}

1. U.S. Department of Transportation, Federal Highway Administration. Status of the Nation's Highways, Bridges, and Transit Conditions and Performance Report, Chapter 7-Capital Investment Scenarios-Highways, 23rd Ed. Available online: https:/ / www.fhwa.dot.gov/policy/23cpr/highlights.cfm\#conditions-and-performance-of-the-system (accessed on 14 September 2021).

2. Montuori, A.; Luzi, G.; Bignami, C.; Gaudiosi, I.; Stramondo, S.; Crosetto, M.; Buongiorno, M.F. The Interferometric Use of Radar Sensors for the Urban Monitoring of Structural Vibrations and Surface Displacements. IEEE J. Sel. Top. Appl. Earth Obs. Remote Sens. 2016, 9, 3761-3776. [CrossRef]

3. Castellano, A.; Fraddosio, A.; Martorano, F.; Mininno, G.; Paparella, F.; Piccioni, M.D. Structural Health Monitoring of a Historic Masonry Bell Tower by Radar Interferometric Measurements. In Proceedings of the IEEE Workshop on Environmental, Energy, and Structural Monitoring Systems (EESMS), Salerno, Italy, 21-22 June 2018; pp. 1-6.

4. Azzara, R.M.; Girardi, M.; Iafolla, V.; Lucchesi, D.M.; Padovani, C.; Pellegrini, D. Ambient vibrations of age-old masonry towers: Results of long-term dynamic monitoring in the historic centre of Lucca. Int. J. Archit. Herit. 2019, 15, 5-21. [CrossRef]

5. Barsocchi, P.; Bartoli, G.; Betti, M.; Girardi, M.; Mammolito, S.; Pellegrini, D.; Zini, G. Wireless sensor networks for continuous structural health monitoring of historic masonry towers. Int. J. Archit. Herit. 2021, 15, 22-44. [CrossRef]

6. Akbar, M.; Akbar, S.J. Dynamic monitoring of bridges: Accelerometer vs microwave radar interferometry (IBIS-S). J. Phys. Conf. Ser. 2021, 1882, 012124.

7. Sivasuriyan, A.; Vijayan, D.S.; Górski, W.; Wodzyński, Ł.; Vaverková, M.D.; Koda, E. Practical Implementation of Structural Health Monitoring in Multi-Story Buildings. Buildings 2021, 11, 263. [CrossRef]

8. Romeo, F.; Di Re, P.; Ciambella, J.; Lofrano, E. Structural analysis and health monitoring of twentieth-century cultural heritage: The Flaminio Stadium in Rome. Smart Struct. Syst. 2021, 27, 285-303.

9. Kot, P.; Muradov, M.; Gkantou, M.; Kamaris, G.S.; Hashim, K.; Yeboah, D. Recent Advancements in Non-Destructive Testing Techniques for Structural Health Monitoring. Appl. Sci. 2021, 11, 2750. [CrossRef]

10. Rice, J.A.; Gu, C.; Li, C.; Guan, S. A radar-based sensor network for bridge displacement measurements. In Sensors and Smart Structures Technologies for Civil, Mechanical, and Aerospace Systems 2012; SPIE: Bellingham, WA, USA, 2012. 
11. Guan, S.; Rice, J.A.; Li, C.; Li, Y.; Wang, G. Structural displacement measurements using DC-coupled radar with an active transponder. Struct. Control Health Monit. 2017, 24, e1909. [CrossRef]

12. Guan, S.; Bridge, J.A.; Li, C.; DeMello, N.J. Smart Radar Sensor Network for Bridge Displacement Monitoring. J. Bridge Eng. 2018, 24, 04018102. [CrossRef]

13. Cardillo, E.; Li, C.; Caddemi, A. Vital sign detection and radar self-motion cancellation through clutter identification. IEEE Trans. Microw. Theory Tech. 2021, 69, 1932-1942. [CrossRef]

14. Li, C.; Lubecke, V.M.; Boric-Lubecke, O.; Lin, J. Sensing of life activities at the human-microwave frontier. IEEE J. Microw. 2021, 1 , 66-78. [CrossRef]

15. Rodriguez, D.; Wang, J.; Li, C. Spoofing attacks to radar motion sensors with portable RF devices. In Proceedings of the IEEE MTT-S Radio and Wireless Symposium (RWS), San Diego, CA, USA, 17-22 January 2021; pp. 73-75.

16. Nallabolu, P.; Zhang, L.; Hong, H.; Li, C. Human Presence Sensing and Gesture Recognition for Smart Home Applications with Moving and Stationary Clutter Suppression Using a 60-GHz Digital Beamforming FMCW Radar. IEEE Access 2021, 9 , 72857-72866. [CrossRef]

17. Feng, C.; Jiang, X.; Jeong, M.G.; Hong, H.; Fu, C.H.; Yang, X.; Wang, E.; Zhu, X.; Liu, X. Multitarget Vital Signs Measurement with Chest Motion Imaging Based on MIMO Radar. IEEE Trans. Microw. Theory Tech. 2021, 69, 4735-4747. [CrossRef]

18. Ren, W.; Qi, F.; Foroughian, F.; Kvelashvili, T.; Liu, Q.; Kilic, O.; Long, T.; Fathy, A.E. Vital Sign Detection in Any Orientation Using a Distributed Radar Network via Modified Independent Component Analysis. IEEE Trans. Microw. Theory Tech. 2021, 69, 4774-4790. [CrossRef]

19. Michel, C.; Keller, S. Advancing Ground-Based Radar Processing for Bridge Infrastructure Monitoring. Sensors 2021, 21, 2172 [CrossRef] [PubMed]

20. Miccinesi, L.; Beni, A.; Pieraccini, M. Multi-monostatic interferometric radar for bridge monitoring. Electronics 2021, $10,247$. [CrossRef]

21. Rodrigues, D.V.Q.; Li, C. A review on low-cost microwave Doppler radar systems for structural health monitoring. Sensors 2021, 21, 2612. [CrossRef]

22. Rodrigues, D.V.Q.; Zuo, D.; Tang, Z.; Wang, J.; Gu, C.; Li, C. Adaptive displacement calibration strategies for field structural health monitoring based on Doppler radars. IEEE Trans. Instrum. Meas. 2020, 69, 7813-7824. [CrossRef]

23. Rodrigues, D.V.Q.; Zuo, D.; Li, C. Wind-induced displacement analysis for a traffic signal structure based on a low-cost Doppler radar array. IEEE Trans. Instrum. Meas. 2021, 70, 6503909. [CrossRef]

24. Rodrigues, D.V.Q.; Tang, Z.; Wang, J.; Zuo, D.; Li, C. Structural Health Monitoring of a Traffic Signal Support Structure Based on 5.8-GHz Doppler Radar with Median Filter and Revised Circle Fitting. In Proceedings of the IEEE Radio and Wireless Symposium (RWS), San Antonio, TX, USA, 26-29 January 2020; pp. 187-190.

25. Sony, S.; Sadhu, A. Multivariate empirical mode decomposition-based structural damage localization using limited sensors. J. Vib. Control 2021, in press. [CrossRef]

26. Hou, S.; Dong, B.; Fan, J.; Wu, G.; Wang, H.; Han, Y.; Zhao, X. Variational Mode Decomposition Based Time-Varying Force Identification of Stay Cables. Appl. Sci. 2021, 11, 1254. [CrossRef]

27. Li, Y.; Lin, J.; Niu, G.; Wu, M.; Wei, X. A Hilbert-Huang Transform-Based Adaptive Fault Detection and Classification Method for Microgrids. Energies 2021, 14, 5040. [CrossRef]

28. Zhao, Y.; Cui, H.; Huo, H.; Nie, Y. Application of Synchrosqueezed Wavelet Transforms for Extraction of the Oscillatory Parameters of Subsynchronous Oscillation in Power Systems. Energies 2018, 11, 1525. [CrossRef]

29. Bernardini, L.; Carnevale, M.; Collina, A. Damage Identification in Warren Truss Bridges by Two Different Time-Frequency Algorithms. Appl. Sci. 2021, 11, 10605. [CrossRef]

30. Eren, C.; Karamzadeh, S.; Kartal, M. Signal Processing Techniques for Human Vital Signs Sensing by Short Range Radar. In Proceedings of the 2020 28th Signal Processing and Communications Applications Conference (SIU), Gaziantep, Turkey, 5-7 October 2020; pp. 1-4.

31. Dong, R.; Cai, D.; Ikuno, S. Motion Capture Data Analysis in the Instantaneous Frequency-Domain Using Hilbert-Huang Transform. Sensors 2020, 20, 6534. [CrossRef] [PubMed]

32. Ricci, R.; Pennacchi, P. Diagnostics of gear faults based on EMD and automatic selection of intrinsic mode functions. Mech. Syst. Signal Process. 2011, 25, 821-838. [CrossRef]

33. Kopsinis, Y.; McLaughlin, S. Investigation and performance enhancement of the empirical mode decomposition method based on a heuristic search optimization approach. IEEE Trans. Signal Process. 2008, 56, 1-13. [CrossRef]

34. Hong, H.; Wang, X.; Tao, Z. Local integral mean-based sifting for empirical mode decomposition. IEEE Signal Process. Lett. 2009, 16, 841-844. [CrossRef]

35. Li, C.; Wang, X.; Tao, Z.; Wang, Q.; Du, S. Extraction of time varying information from noisy signals: An approach based on the empirical mode decomposition. Mech. Syst. Signal Process. 2011, 25, 812-820. [CrossRef]

36. Daubechies, I.; Lu, J.; Wu, H.-T. Synchrosqueezed wavelet transforms: An empirical mode decomposition-like tool. Appl. Comput. Harmon. Anal. 2011, 30, 243-261. [CrossRef]

37. Tary, J.B.; Baan, M.V.D. Attenuation estimation using high resolution time-frequency transforms. Digit. Signal Process. 2017, 60, 46-55. [CrossRef] 
38. Auger, F.; Flandrin, P. Time-frequency reassignment and synchrosqueezing: An overview. IEEE Signal Process. Mag. 2013, 30, 32-41. [CrossRef]

39. Qin, S.; Zhou, S.; Zhu, T.; Zhu, S.; Li, J.; Zheng, Z.; Qin, S.; Pan, C.; Tang, J. Sinusoidal Noise Removal in PD Measurement Based on Synchrosqueezing Transform and Singular Spectrum Analysis. Energies 2021, 14, 7967. [CrossRef]

40. Chen, H.; Lu, L.; Xu, D.; Kang, J.; Chen, X. The Synchrosqueezing Algorithm Based on Generalized S-transform for High-Precision Time-Frequency Analysis. Appl. Sci. 2017, 7, 769. [CrossRef]

41. Hu, Y.; Tu, X.; Li, F.; Meng, G. Joint High-Order Synchrosqueezing Transform and Multi-Taper Empirical Wavelet Transform for Fault Diagnosis of Wind Turbine Planetary Gearbox under Nonstationary Conditions. Sensors 2018, 18, 150. [CrossRef] [PubMed]

42. Daubechies, I.; Wang, Y.G.; Wu, H.-T. Conceft: Concentration of frequency and time via a multitapered synchrosqueezed transform. Philos. Trans. R. Soc. A 2016, 374, 20150193. [CrossRef]

43. Bruni, V.; Tartaglione, M.; Vitulano, D. On the time-frequency reassignment of interfering modes in multicomponent FM signals. In Proceedings of the 26th European Signal Processing Conference (EUSIPCO), Rome, Italy, 3-7 September 2018; pp. 722-726.

44. Gençay, R.; Selçuk, F.; Whitcher, B.J. An Introduction to Wavelets and Other Filtering Methods in Finance and Economics; Academic Press: San Diego, CA, USA, 2001.

45. Lilly, J.M.; Olhede, S.C. Higher-Order Properties of Analytic Wavelets. IEEE Trans. Signal Process. 2009, 57, 146-160. [CrossRef]

46. Deléchelle, E.; Lemoine, J.; Niang, O. Empirical mode decomposition: An analytical approach for sifting process. IEEE Signal Process. Lett. 2005, 12, 764-767. [CrossRef]

47. Park, B.-K.; Boric-Lubecke, O.; Lubecke, V.M. Arctangent demodulation with DC offset compensation in quadrature Doppler radar receiver systems. IEEE Trans. Microw. Theory Tech. 2007, 55, 1073-1079. [CrossRef]

48. Guan, S.; Rice, J.A.; Li, C.; Gu, C. Automated DC offset calibration strategy for structural health monitoring based on portable CW radar sensor. IEEE Trans. Instrum. Meas. 2014, 63, 3111-3118. [CrossRef]

49. Percival, D.B.; Walden, A.T. Wavelet Methods for Time Series Analysis. Cambridge Series in Statistical and Probabilistic Mathematics; Cambridge University Press: New York, NY, USA, 2000.

50. Percival, D.B. Analysis of Geophysical Time Series Using Discrete Wavelet Transforms: An Overview. In Nonlinear Time Series Analysis in the Geosciences; Lecture Notes in Earth Sciences; Donner, R.V., Barbosa, S.M., Eds.; Springer: Berlin/Heidelberg, Germany, 2008; Volume 112.

51. Polanco-Martínez, J.M.; Abadie, L.M. Analyzing Crude Oil Spot Price Dynamics versus Long Term Future Prices: A Wavelet Analysis Approach. Energies 2016, 9, 1089. [CrossRef]

52. Barroso-García, V.; Gutiérrez-Tobal, G.C.; Gozal, D.; Vaquerizo-Villar, F.; Álvarez, D.; del Campo, F.; Kheirandish-Gozal, L.; Hornero, R. Wavelet Analysis of Overnight Airflow to Detect Obstructive Sleep Apnea in Children. Sensors 2021, $21,1491$. [CrossRef] [PubMed]

53. Shams, M.A.; Anis, H.I.; El-Shahat, M. Denoising of Heavily Contaminated Partial Discharge Signals in High-Voltage Cables Using Maximal Overlap Discrete Wavelet Transform. Energies 2021, 14, 6540. [CrossRef]

54. Yang, D.-M. The Detection of Motor Bearing Fault with Maximal Overlap Discrete Wavelet Packet Transform and Teager Energy Adaptive Spectral Kurtosis. Sensors 2021, 21, 6895. [CrossRef] [PubMed]

55. Niven, I.; Zuckerman, H.S.; Montgomery, H.L. An Introduction to the Theory of Numbers; John Wiley \& Sons, Inc.: Hoboken, NJ, USA, 1991.

56. Hardy, G.H.; Wright, E.M. An Introduction to the Theory of Numbers; Oxford University Press: New York, NY, USA, 2008.

57. Lacerda, J.C.A. Praticando a Aritmética; XYZ Editora: Rio de Janeiro, Brazil, 2018. 